\title{
ON THE SAUER-XU FORMULA FOR THE ERROR IN MULTIVARIATE POLYNOMIAL INTERPOLATION
}

\author{
CARL DE BOOR
}

\begin{abstract}
Use of a new notion of multivariate divided difference leads to a short proof of a formula by Sauer and $\mathrm{Xu}$ for the error in interpolation, by polynomials of total degree $\leq n$ in $d$ variables, at a 'correct' point set.
\end{abstract}

It is the purpose of this note to give a short proof of a remarkable formula for the error in polynomial interpolation given in [3].

In [3], Sauer and Xu consider interpolation from the space $\Pi_{n}\left(\mathbf{R}^{d}\right)$ of $d$-variate polynomials of degree $\leq n$ at a point set $\mathcal{X}$ which is correct for it in the sense that, for an arbitrary $g$, there is exactly one $p \in \Pi_{n}\left(\mathbf{R}^{d}\right)$, denoted here by

$$
P_{n} g
$$

which agrees with $g$ on $\mathcal{X}$. Such a correct point set can, as Sauer and Xu point out, be partitioned into subsets

$x^{(i)}:=\left\{x_{r}^{(i)}: r=1, \ldots, r_{i}^{d}:=\operatorname{dim} \Pi_{i}-\operatorname{dim} \Pi_{i-1}=\left(\begin{array}{c}i-1+d \\ i\end{array}\right)\right\}, \quad i=0,1, \ldots, n$, in such a way that, for each $j \leq n$, polynomial interpolation from $\Pi_{j}$ at the points in

$$
x^{(\leq j)}:=x^{(0)} \cup \cdots \cup x^{(j)}
$$

is uniquely possible. They denote the corresponding Lagrange polynomial in $\Pi_{j}$ associated with the point $x_{r}^{(j)}$ by

$$
p_{r}^{[j]}
$$

i.e., $p_{r}^{[j]}$ is the unique element of $\Pi_{j}$ which satisfies

$$
p_{r}^{[j]}\left(x_{s}^{(i)}\right)=\delta_{j i} \delta_{r s}, \quad s=1, \ldots, r_{i}^{d} ; i=0, \ldots, j,
$$

but is called a Newton polynomial in [3], probably because any $p \in \Pi_{j}$ which vanishes on $x^{(<j)}$ can be written in the form

$$
p=\sum_{r=1}^{r_{j}^{d}} p_{r}^{[j]} p\left(x_{r}^{(j)}\right) .
$$

Received by the editor May 8, 1995 .

1991 Mathematics Subject Classification. Primary 41A05, 41A10, 65D05.

Key words and phrases. Polynomials, multivariate, interpolation, error, remainder formula, divided difference.

This work was supported by the NSF grant DMS-9224748, by the US-Israel Binational Science Foundation under Grant No. 90-00220, and by ARO under Grant No. DAA H04-95-1-0089. 
Let $E_{j} g$ denote the error in the polynomial interpolant from $\Pi_{j}$ to $g$, i.e.,

$$
g=P_{j} g+E_{j} g
$$

Sauer and $\mathrm{Xu}$ prove that (in roughly the same notation, except for a slight reordering and the use of a divided difference here)

$$
\left(E_{j} g\right)(x)=\sum_{r=1}^{r_{j}^{d}} p_{r}^{[j]}(x) \sum_{\mu \in \Lambda_{r}^{(j)}} c_{\mu}\left[x_{\mu}, x ; \Delta x_{\mu}, x-x_{r}^{(j)}\right] g,
$$

with the following definition of the various quantities appearing here:

$$
\begin{gathered}
\Lambda_{r}^{(j)}:=\left\{\mu \in \times_{i=0}^{j}\left\{1, \ldots, r_{i}^{d}\right\}: \mu_{j}=r\right\} ; \\
c_{\mu}:=\prod_{i=0}^{j-1} p_{\mu_{i}}^{[i]}\left(x_{\mu_{i+1}}^{(i+1)}\right) ; \\
x_{\mu}:=\left(x_{\mu_{i}}^{(i)}: i=0, \ldots, j\right), \quad \Delta x_{\mu}:=\left(x_{\mu_{i+1}}^{(i+1)}-x_{\mu_{i}}^{(i)}: i=0, \ldots, j-1\right) ;
\end{gathered}
$$

and, finally, $\left[t_{0}, \ldots, t_{j} ; \xi_{1}, \ldots, \xi_{j}\right]$ is the $j$ th divided difference introduced in [1], i.e.,

$$
\left[t_{0}, \ldots, t_{j} ; \xi_{1}, \ldots, \xi_{j}\right] g:=\int_{\left[t_{0}, \ldots, t_{j}\right]} D_{\xi_{1}} \cdots D_{\xi_{j}} g
$$

with

$$
f \mapsto \int_{\left[t_{0}, \ldots, t_{j}\right]} f:=\int_{0}^{1} \int_{0}^{s_{1}} \cdots \int_{0}^{s_{j-1}} f\left(t_{0}+s_{1} \nabla t_{1}+\cdots+s_{j} \nabla t_{j}\right) \mathrm{d} s_{j} \cdots \mathrm{d} s_{1}
$$

the linear functional which was used to such advantage by Micchelli in his analysis of Kergin interpolation and the simplex spline, and which has been dubbed by him in [2] the divided difference functional for $\mathbf{R}^{d}$. The only facts about the $j$ th divided difference (3) needed here are that it is symmetric in the points $t_{0}, \ldots, t_{j}$, and is symmetric and linear in the directions $\xi_{1}, \ldots, \xi_{j}$ (which is obvious), and that, for an arbitrary point sequence $T$, points $a, b$, and arbitrary direction sequence $\Xi$ (with $\# T=\# \Xi)$,

$$
[T, a, b ; \Xi, a-b]=[T, a ; \Xi]-[T, b ; \Xi]
$$

which can be verified directly (see [1]).

The short proof of (2) about to be given here is by induction (as is the proof in [3]). For $j=0,(2)$ is the special case $T=()=\Xi, a=x, b=x_{1}^{(0)}$ of (4). Assuming (2) to hold for $j=n$, we observe that

$$
\left(E_{n} g\right)(x)=G_{n}(x, x)
$$


with

$$
G_{n}(x, y):=\sum_{r=1}^{r_{n}^{d}} p_{r}^{[n]}(x) \sum_{\mu \in \Lambda_{r}^{(n)}} c_{\mu}\left[x_{\mu}, y ; \Delta x_{\mu}, x-x_{r}^{(n)}\right] g .
$$

Now note that, for arbitrary $y, G_{n}(\cdot, y)$ is a polynomial of degree $\leq n+1$ (since the $p_{r}^{[n]}$ are in $\Pi_{n}$ while $x \mapsto[T ; \Xi, x] g$ is a linear (scalar-valued) function), and $G_{n}(\cdot, y)$ vanishes at every $x_{r}^{(n)}$, hence, as in $(1), G_{n}(\cdot, y)$ is writeable as

$$
G_{n}(\cdot, y)=\sum_{s=1}^{r_{n+1}^{d}} p_{s}^{[n+1]} G_{n}\left(x_{s}^{(n+1)}, y\right)
$$

On the other hand, since $g=P_{n} g+E_{n} g$, the function

$$
P_{n} g+\sum_{s=1}^{r_{n+1}^{d}} p_{s}^{[n+1]}\left(E_{n} g\right)\left(x_{s}^{(n+1)}\right)
$$

agrees with $g$ at $x^{(\leq n+1)}$ and is in $\Pi_{n+1}$, hence must equal $P_{n+1} g$. Therefore,

$$
\begin{aligned}
E_{n+1} g & =E_{n} g-\sum_{s} p_{s}^{[n+1]}\left(E_{n} g\right)\left(x_{s}^{(n+1)}\right) \\
& =\sum_{s} p_{s}^{[n+1]}\left(G_{n}\left(x_{s}^{(n+1)}, \cdot\right)-G_{n}\left(x_{s}^{(n+1)}, x_{s}^{(n+1)}\right)\right),
\end{aligned}
$$

and this implies (2) for $j=n+1$ since

$$
G_{n}\left(x_{s}^{(n+1)}, \cdot\right)-G_{n}\left(x_{s}^{(n+1)}, x_{s}^{(n+1)}\right)=\sum_{r=1}^{r_{n}^{d}} p_{r}^{[n]}\left(x_{s}^{(n+1)}\right) \sum_{\mu \in \Lambda_{r}^{(n)}} c_{\mu} d_{\mu, r} g
$$

with

$$
\begin{aligned}
d_{\mu, r}:=\left[x_{\mu}, \cdot ; \Delta x_{\mu}, x_{s}^{(n+1)}\right. & \left.-x_{r}^{(n)}\right]-\left[x_{\mu}, x_{s}^{(n+1)} ; \Delta x_{\mu}, x_{s}^{(n+1)}-x_{r}^{(n)}\right] \\
& =\left[x_{\mu}, x_{s}^{(n+1)}, \cdot ; \Delta x_{\mu}, x_{s}^{(n+1)}-x_{r}^{(n)}, \cdot-x_{s}^{(n+1)}\right],
\end{aligned}
$$

by (4).

It follows that

$$
P_{n} g=\sum_{j=0}^{n} \sum_{r=1}^{r_{j}^{d}} p_{r}^{[j]}\left(E_{j-1} g\right)\left(x_{r}^{(j)}\right)
$$

with

$$
E_{-1} g:=g .
$$

Since $\left(p_{r}^{[j]}: r=1, \ldots, r_{j}^{d} ; j=0,1, \ldots\right)$ is linearly independent, it follows that, for any $j,[x ;] E_{j}$ is a linear combination of the linear functionals $[x ;]$ and $\left[x_{r}^{(i)} ;\right], r=$ $1, \ldots, r_{i}^{d} ; i=0, \ldots, j$. However, its constituents, i.e., the $j$ th divided differences

$$
\left[x_{\mu}, x ; \Delta x_{\mu}, x-x_{\mu_{j}}^{(j)}\right]
$$

by themselves, are not necessarily such linear combinations, as the simple example

$$
\left[0, \mathbf{i}_{1}, \mathbf{i}_{1}+\mathbf{i}_{2} ; \mathbf{i}_{1}, \mathbf{i}_{2}\right]
$$

with $\mathbf{i}_{j}$ the $j$ th unit vector, readily shows. 


\section{REFERENCES}

1. Carl de Boor, A multivariate divided difference, Approximation Theory VIII, Academic Press, New York, 1995, pp. 87-96.

2. C. A.Micchelli, On a numerically efficient method for computing multivariate B-splines, Multivariate Approximation Theory, Birkhäuser, Basel, 1979, pp. 211-248. MR 81g:65017

3. T.Sauer and Yuan Xu, On multivariate Lagrange interpolation, Math. Comp. 64 (1995), 1147-1170. MR 95j:41051

Computer Sciences Department, University of Wisconsin-Madison, 1210 W. Dayton St., Madison, Wisconsin 53706

E-mail address: deboor@cs.wisc.edu 